\title{
Effects of long-term clarithromycin treatment on lavage- fluid markers of inflammation in chronic rhinosinusitis
}

Anders Cervin ${ }^{1,2,4} \mathrm{MD}$, Ben Wallwork ${ }^{1,2} \mathrm{MD}$, Alan Mackay-Sim ${ }^{1} \mathrm{PhD}$, William B. Coman ${ }^{2,3}$ MD, Lennart Greiff ${ }^{1,2,4}$ MD.

${ }^{1}$ National Centre for Adult Stem Cell Research, Griffith University, Brisbane, Australia.

${ }^{2}$ Department of Otorhinolaryngology, Princess Alexandra Hospital, Brisbane, Australia. ${ }^{3}$ Department of Otorhinolaryngology, University of Queensland, Brisbane, Australia. ${ }^{4}$ Department of Otorhinolaryngology, Lund University Hospital, Lund, Sweden.

Correspondence: Anders Cervin

Department of Otorhinolaryngology

Helsingborg Hospital

SE-25 187 Helsingborg

Sweden

Tel: $\quad+46-42-4061000$

Fax: $\quad+46-46-2110968$

E-mail: $\quad$ anders.cervin@med.lu.se

Short title: Effects of clarithromycin in chronic rhinosinusitis. 


\section{Summary}

Macrolides can be clinically effective in chronic rhinosinusitis (CRS). However, little is known about how these drugs affect pathophysiological features of CRS in vivo. In the present study, patients with CRS were subjected to long-term treatment with clarithromycin. Nasal lavages with and without histamine (40 and $400 \mu \mathrm{g} / \mathrm{ml}$ ) were carried out prior to and late into the treatment period. Histamine was included as a tool to produce plasma exudation, a process known to move free cellular products from the mucosal tissue into the airway lumen thereby enriching nasal surface liquids with such products. IL-8, MPO, ECP, $\alpha_{2}$-macroglobulin, and fucose were monitored as indices of pro-inflammatory cytokine production, neutrophil and eosinophil granulocyte activities, plasma exudation, and mucinous secretion, respectively. Clarithromycin reduced the lavage fluid levels of IL-8 at the low-dose histamine observation $(\mathrm{p}<0.001)$. There was a trend towards reduced MPO by the treatment, whereas ECP was significantly reduced at the low-dose histamine observation $(\mathrm{p}<0.05)$. Alpha 2 -Macroglobulin was reduced by clarithromycin (saline lavages) $(\mathrm{p}=0.05)$, whereas fucose was unaffected. The exudative responsiveness to high-dose histamine was significantly reduced by the treatment $(\mathrm{p}<0.05)$. Furthermore, significantly lower levels of fucose were observed at the lowdose histamine observation $(\mathrm{p}<0.01)$. We conclude that long-term clarithromycin treatment likely exerts anti-inflammatory effect in CRS.

Key words: chronic rhinosinusitis, ECP, fucose, IL-8, $\alpha_{2}$-macroglobulin, myeloperoxidase. 


\section{Introduction}

Macrolides are important chemotherapeutics in the treatment of infections. Over the last decade, there has been an interest in the possibility that these drugs may be effective also in non-infectious chronic rhinosinusitis (CRS) (Ichimura, et al. 1996; Cervin, et al. 2002). Recently, we demonstrated a symptom-reducing effect of roxithromycin in CRS in the first placebo-controlled trial involving macrolides. Observations in vitro have shown that macrolides may inhibit a potentially pro-inflammatory airway epithelial cell production of interleukin-8 (IL-8) in (Suzuki, et al. 1999; Wallwork, et al. 2002; Wallwork, et al. 2006), a cytokine associated with neutrophil activity (Suzuki, et al. 1997). Accordingly, it may be hypothesised that macrolides exert an anti-inflammatory effect in CRS by reducing IL-8. However, with few exceptions, little is as yet known about how macrolide treatment affects nasal inflammatory processes associated with CRS in vivo (Yamada, et al. 2000; MacLeod, et al. 2001).

CRS is characterised by nasal mucosal infiltration of neutrophil granulocytes (neutrophils) and by increased nasal lavage fluid levels of the neutrophil product myeloperoxidase (MPO) (Hamilos, et al. 1993; Demoly, et al. 1997). Furthermore, increased gene expression and nasal mucosal surface levels of IL-8 are reported (Takeuchi, et al. 1995; Rudack, et al. 1998). Taken together, these observations indicate that a neutrophil inflammatory potential characterises CRS. However, increased numbers of eosinophil granulocytes (eosinophils) (Hamilos, et al. 1993) and increased nasal lavage fluid levels of granulocyte macrophage colony-stimulating factor (GM-CSF), both features associated with allergic conditions, are also reported in CRS. Although an increased eosinophil activity in CRS may suggest a presence of atopy, a mixed granulocyte response in non-atopic CRS may not be surprising. For example, it has been shown that common cold may be characterised by such a response (Greiff, et al. 2002).

In this open study, we have examined whether or not long-term treatment with clarithromycin affects inflammatory features in CRS. We have carried out nasal lavages prior to and late into the treatment period and monitored IL-8, MPO, and eosinophil cationic protein (ECP), reflecting pro-inflammatory cytokine production and granulocyte activity. In addition, we have explored the possibility that clarithromycin may affect end-organ activities potentially associated with CRS. Thus, we have 
monitored $\alpha_{2}$-macroglobulin and fucose as indices of plasma exudation and mucinous secretion, respectively. Finally, we have carried out lavages with histamine: these have been included as a tool to produce extravasation and luminal entry of plasma, a process known to move free cellular products from the lamina propria into the airway lumen thereby enriching nasal surface liquids with products such as IL-8, MPO, and ECP. Furthermore, the histamine lavages have the capacity to allow for an estimation of the exudative and secretory responsiveness of the nasal mucosa (Greiff, et al. 2005). 


\section{Methods}

\section{Study design}

Patients with CRS received treatment with clarithromycin $250 \mathrm{mg}$ once daily for twelve weeks in an open study design. Nasal lavages with and without histamine were carried out before and ten weeks into the treatment period. Lavage fluid levels of IL-8, MPO, ECP, $\alpha_{2}$-macroglobulin, and fucose, were measured as indices of pro-inflammatory cytokine production, neutrophil activity, eosinophil activity, plasma exudation, and mucinous secretion, respectively. The patients were asked whether or not they improved during the course of the study.

\section{Subjects}

Subjects were recruited from ENT-Consultants working within the catchment area of Princess Alexandra Hospital, Brisbane, Australia. The patients were subjected to a physical examination including a nasal endoscopy, ensuring patent ostia of the maxillary sinus. Furthermore, a nasal/nasopharyngeal culture, IgE and RAST for common allergens were performed. Inclusion criteria were a history of CRS with prior sinus surgery followed by persistent symptoms of nasal discharge, nasal stuffiness, and/or sinus related facial pain (according to the criteria of the American Academy of Otolaryngology, Head and Neck Surgery). Exclusion criteria were a diagnosis of cystic fibrosis, primary ciliary dyskinesia, fungal sinusitis, and positive nasal/nasopharyngeal cultures. Patients on drug treatment were excluded with the exception of the occasional use of nasal decongestants. Accordingly, glucocorticosteroid treatment was not allowed and any such treatment was withdrawn at least four-weeks before study entry. The study was approved by the Ethics Committee at Princess Alexandra Hospital and informed consent was obtained.

\section{Drug treatment}

Treatment with $250 \mathrm{mg}$ clarithromycin once daily was administered in an open, nonplacebo controlled design. The treatment was continued for twelve weeks and clinical assessments and nasal lavages were carried out before and late into the treatment period.

\section{Nasal lavage}


A pool-device was used for saline lavage and for concomitant histamine challenge and lavage of the nasal mucosa (Greiff, et al. 1990). In the present study, the volume of the nasal pool-fluid was $15 \mathrm{ml}$ and the duration of each lavage five minutes. Isotonic saline and histamine (40 and $400 \mu \mathrm{g} / \mathrm{ml}$ ) in isotonic saline were administered in sequence with five-minute intervals. The lavage-fluids were centrifuged (325 g, $10 \mathrm{~min}, 4^{\circ} \mathrm{C}$ ) and samples were obtained from the supernatant and frozen $\left(-20^{\circ} \mathrm{C}\right)$ awaiting analysis.

\section{Analysis}

The lavage fluid samples were processed by ultrasonication. $\alpha_{2}$-Macroglobulin was measured using a radioimmunoassay sensitive to $7.8 \mathrm{ng} / \mathrm{ml}$. Rabbit anti-human $\alpha_{2}$ macroglobulin (Dakopatts, Copenhagen, Denmark) was used as anti-serum and human serum (Behringwerke Diagnostica, Marburg, Germany) as standard. Human $\alpha_{2}$-macroglobulin (Cappel-Organon, Turnhout, Belgium) was iodinated using the lactoperoxidase method. Tracer and standard or sample was mixed with anti-serum before adding goat anti-rabbit anti-serum (AstraZeneca, Lund, Sweden). The bound fraction was measured using a gamma counter (Pharmacia Diagnostics, Uppsala, Sweden). The intra- and inter-assay coefficients of variation were 3.8-6.0\% and 3.1-7.2\%, respectively. Fucose was measured using a parallel ligand-exchange chromatography in combination with post-column derivatisation and fluorescence detection (Freney, et al. 2001). MPO was measured using an ELISA (R\&D Systems, London, UK). The detection level of the assay was $<8 \mathrm{ng} / \mathrm{ml}$. ECP was measured by a fluoroimmunoassay (Pharmacia Diagnostics, Uppsala, Sweden). The detection level of the ECP assay was $<2 \mathrm{ng} / \mathrm{ml}$. IL8 was measured using an ELISA (R\&D Systems, London, UK). The detection level of the ECP assay was $<31 \mathrm{pg} / \mathrm{ml}$.

\section{Statistics}

Differences in nasal lavage fluid of levels of $\alpha_{2}$-macroglobulin, fucose, MPO, ECP, and IL-8 between observations prior to and after treatment were examined using the Wilcoxon signed rank test. Correlations between nasal lavage fluid levels of IL-8 and ECP were tested for using the Spearman rank correlation test. P-values of 0.05 or less were considered statistically significant. Data are presented as mean \pm SEM. 


\section{Results}

Thirty patients were recruited to the study ( 21 women, 9 men). The mean age was $46 \pm 10$ years (range $25-63$ ). The duration of disease was 18 \pm 15 years (range 1-60) and the number of surgical sinus procedures $3 \pm 2$ (range 1-7). Five patients failed to follow the protocol. Of the remaining 25, 14 reported improvement during the treatment (data not shown).

The levels of IL-8 were unaffected by the histamine-lavages carried out before treatment (Fig. 1A). Clarithromycin reduced the saline lavage levels of IL-8, but this effect failed to reach statistical significance $(p=0.082)$. In contrast, IL-8 was significantly reduced by clarithromycin at the low-dose histamine-observation $(\mathrm{p}<0.001)$.

There was a trend towards reduced levels of MPO following clarithromycin treatment at the saline and low-dose histamine observations (Fig. 1B), but this failed to reach statistical significance. A similar reduction was also observed for ECP (Fig. 1C), and this effect reached statistical significance at the low-dose histamine observation $(\mathrm{p}<0.05)$.

The saline lavage levels of $\alpha_{2}$-macroglobulin were reduced following clarithromycin treatment $(\mathrm{p}=0.05)$ (Fig. 2A). As would be expected, histamine produced significant plasma exudation prior to and following clarithromycin treatment $(\mathrm{p}<0.05)$. However, the exudative responsiveness to high-dose histamine was significantly reduced by clarithromycin $(\mathrm{p}<0.05)$.

Clarithromycin did not affect saline lavage levels of fucose (Fig. 2B). High dose histamine produced significantly increased fucose levels $(\mathrm{p}<0.05)$. This effect was reduced by clarithromycin, but the change failed to reach statistical significance $(p<0.093)$. However, significantly lower levels of fucose were observed at the low-dose histamine observation $(\mathrm{p}<0.01)$. 


\section{Discussion}

In the present study, we showed that long-term treatment with clarithromycin in CRS was associated with reduced nasal mucosal output of IL-8. Furthermore, the output of granulocyte products, notably ECP, was reduced. The use of histamine-lavages, employed to produce extravasation and luminal entry of plasma and thereby enriching mucosal surface levels with cellular products of the mucosal tissue, was critical in order to detect the changes. In terms of end-organ responses of the nasal mucosa, the study demonstrated a reduction in nasal mucosal output of plasma ( $\alpha_{2}$-macroglobulin) following clarithromycin treatment, but no effects on baseline (saline lavage) mucinous secretion (fucose). The ability of histamine to produce plasma exudation, i.e., the exudative responsiveness of the mucosa, and mucinous secretion, i.e., the secretory responsiveness, was significantly reduced by clarithromycin. Our findings suggest that clarithromycin exerts anti-inflammatory effect in CRS.

In a recent study, focusing on symptoms and clinical signs of CRS, we originally demonstrated that treatment with roxithromycin was superior to placebo (Wallwork, et al. 2006). Thereby, we confirmed a series of preceding reports suggesting this particular effect of macrolides (Ichimura, et al. 1996; Cervin, et al. 2002). In contrast to studies with focus on symptoms of disease, we regarded the present non-placebo controlled design as justified since it focused exclusively on objective markers of inflammation. However, given the design, the study material was analysed as a whole. The drawback of this strategy was that a mixture of what might be regarded as clinical responders and failures were analysed together, which likely impaired the possibility to detect treatment specific changes. A second disadvantage of the design was that it did not comprise control subjects. Accordingly, characteristics of CRS in terms of which pathophysiological features that characterized the present patients might not be deduced. However, based on the treatment effects (below), and in agreement with previous reports (Hamilos, et al. 1993; Demoly, et al. 1997; Takeuchi \& Ichimura 1997; Rudack, et al. 1998) our findings suggest that the present CRS patients feature an inflammatory activity.

The process of extravasation, lamina propria distribution, and luminal entry of bulk plasma is a key innate airway defence mechanism and a feature of airway inflammation. 
When induced by histamine-lavages, this process moves non-plasma tissue solutes (released from inflammatory cells) from the lamina propria into the airway lumen (Greiff, et al. 2003). The advantage of this tool is that low-grade inflammatory activity can be detected in the airway lumen when it would not be detectable by plain saline lavages. In the present study, we confirmed the exudative effect of histamine-lavages. The findings prior to treatment indicated that the histamine-induced luminal entry of plasma moved free IL-8 from the tissue into the airway lumen, suggesting increased IL8 activity in CRS. In contrast, this was not seen following clarithromycin treatment, suggesting an anti-inflammatory effect of the intervention. The fact that this was not observed at high-dose histamine observations was not surprising because of the limited tissue-pool of IL-8 and since the first histamine-lavage likely depleted the tissue of this cytokine. We may not, given the present design, discuss in detail which pathophysiological features that characterise CRS, but we can conclude that clarithromycin reduced the levels of the pro-inflammatory cytokine IL-8.

Lavage fluid analyses of IL-8 and MPO were included in the present study as indices of pro-inflammatory cytokine production and neutrophil activity, respectively. In that context, IL-8 was considered to be a neutrophil chemokine affected by macrolides in vitro (Suzuki, et al. 1997; Wallwork, et al. 2002). In the present CRS patients, we demonstrated that a clarithromycin inhibited airway release of IL-8 in vivo. Our observation (in CRS) was in agreement with previous reports by Yamada et al. (Yamada, et al. 2000), suggesting a similar effect of this class of drugs in nasal polyposis, and by MacLeod et al. (MacLeod, et al. 2001) focusing on chronic sinusitis/persistent maxillary sinus inflammation. In contrast to IL-8, we observed no significant effect of the treatment on nasal lavage fluid levels of MPO despite the fact the levels were reduced by 48 and $55 \%$ in saline and low-dose histamine-lavages, respectively. Our failure to detect significant changes in MPO might reflect that the baseline levels of this mediator were high and variable already at baseline conditions. Taken together, our data was in agreement with the hypothesis that macrolides exert an IL-8 reducing effect in CRS, but we could not convincingly show that this effect lead to reduced neutrophil activity. We suggest that future studies on the effects of macrolides in CRS shall employ lavage as well as tissue indices in order to monitor neutrophil presence and activity more accurately. 
In the present study, the levels of ECP in saline lavages obtained following clarithromycin treatment were reduced (c.f. observations before treatment), but this $56 \%$ reduction failed to reach statistical significance. In contrast, significance was achieved at the low-dose histamine observation. Adding to previous reports on presence of eosinophils in CRS (Hamilos, et al. 1993), our ECP observations suggested the possibility that the present CRS patients were characterised by increased eosinophil activity. Furthermore, our findings indicated that this feature of CRS was reduced by clarithromycin treatment. The notion that CRS could be characterised by increased eosinophil activity might not be surprising since many inflammatory processes are characterized by mixed granulocyte responses: for example, common cold may be associated with increased neutrophil and eosinophil activity (Greiff, et al. 2002). Moreover, the neutrophil chemokine IL-8 may under certain circumstances act also as an eosinophil chemokine (Warringa, et al. 1993; Erger \& Casale 1998). In the present study, this possibility was suggested by a statistically significant positive correlation between nasal lavage fluid levels of IL-8 and ECP (rho=0.655, p<0.05, 150 paired observations). Accordingly, it may be hypothesised that the present reduction in nasal mucosal output of ECP was secondary to the reduction in IL-8.

Plasma exudation is a key feature of airway inflammation. In allergic rhinitis, this process is attenuated by topical corticosteroids, indicating the anti-inflammatory properties of this class of drugs. The effect likely reflects actions on inflammatory cells that fuel inflammation rather than vascular anti-permeability effects (Greiff, et al. 2003). In the present study, we monitored $\alpha_{2}$-macroglobulin as index of plasma exudation and the levels of this non-secreted plasma protein were significantly lower in saline lavages obtained following clarithromycin treatment compared with prior to treatment. The finding indicates that clarithromycin exerts an anti-inflammatory effect in CRS. In the present study, clarithromycin also reduced the exudative responsiveness to histamine. The development of an increased exudative responsiveness is a key feature of airway inflammation (Greiff, et al. 1994; Svensson, et al. 1995) and in allergic rhinitis it is particularly sensitive to corticosteroid treatment (Meyer, et al. 1999). Whether or not the present CRS subjects featured an increased exudative responsiveness could be deduced from the present study. However, the observation that clarithromycin significantly reduced the exudative responsiveness to histamine is in agreement with the view that clarithromycin exerts an anti-inflammatory effect in CRS. 
Secretion is a mucosal defence mechanism (Rogers 1994). Moreover, secretion is a pathological event in airway disease (Rogers 1994). In the present study, reflecting that the CRS patients suffered from rhinorrhea, we monitored treatment-effects of clarithromycin on nasal lavage fluid levels of fucose (6-deoxy galactose): a sugar moiety of the mucin molecule. Fucose is localized to goblet cells and airway glands (Mariassy, et al. 1988; Castells, et al. 1991). Accordingly, we forwarded fucose as a global marker of mucinous secretions (Freney, et al. 2001; Greiff, et al. 2005). We demonstrated that several topical challenges, including histamine, produced increased nasal mucosal output of fucose (Greiff, et al. 2005). In agreement, the present nasal exposure to histamine increased significantly the lavage fluid levels of fucose. This secretory responsiveness to histamine was reduced by the clarithromycin treatment. In contrast, baseline levels of fucose were unaffected by the treatment. Taken together, the present data suggest that CRS may feature a secretory hyperresponsiveness to histamine and indicate that this feature is reduced by the clarithromycin treatment.

We conclude that long-term treatment with clarithromycin exerts an anti-inflammatory effect in CRS. The conclusion is substantiated by the demonstration of reduced levels inflammatory products and $\alpha_{2}$-macroglobulin (a marker of plasma exudation) following treatment. Furthermore, it is suggested by the attenuated responsiveness of the nasal mucosa to histamine. 


\section{Acknowledgements}

A. Cervin was supported by the Garnett Passe \& Rodney Williams Memorial Foundation, the Swedish Research Council, and the Swedish Society of Medicine. B. Wallwork, A. Mackay-Sim, and W. B. Coman were supported by the Garnett Passe \& Rodney Williams Memorial Foundation. L. Greiff was supported by the Swedish Research Council, the Swedish Society of Medicine, and the Swedish Otorhinolaryngological Society. 


\section{Figures}

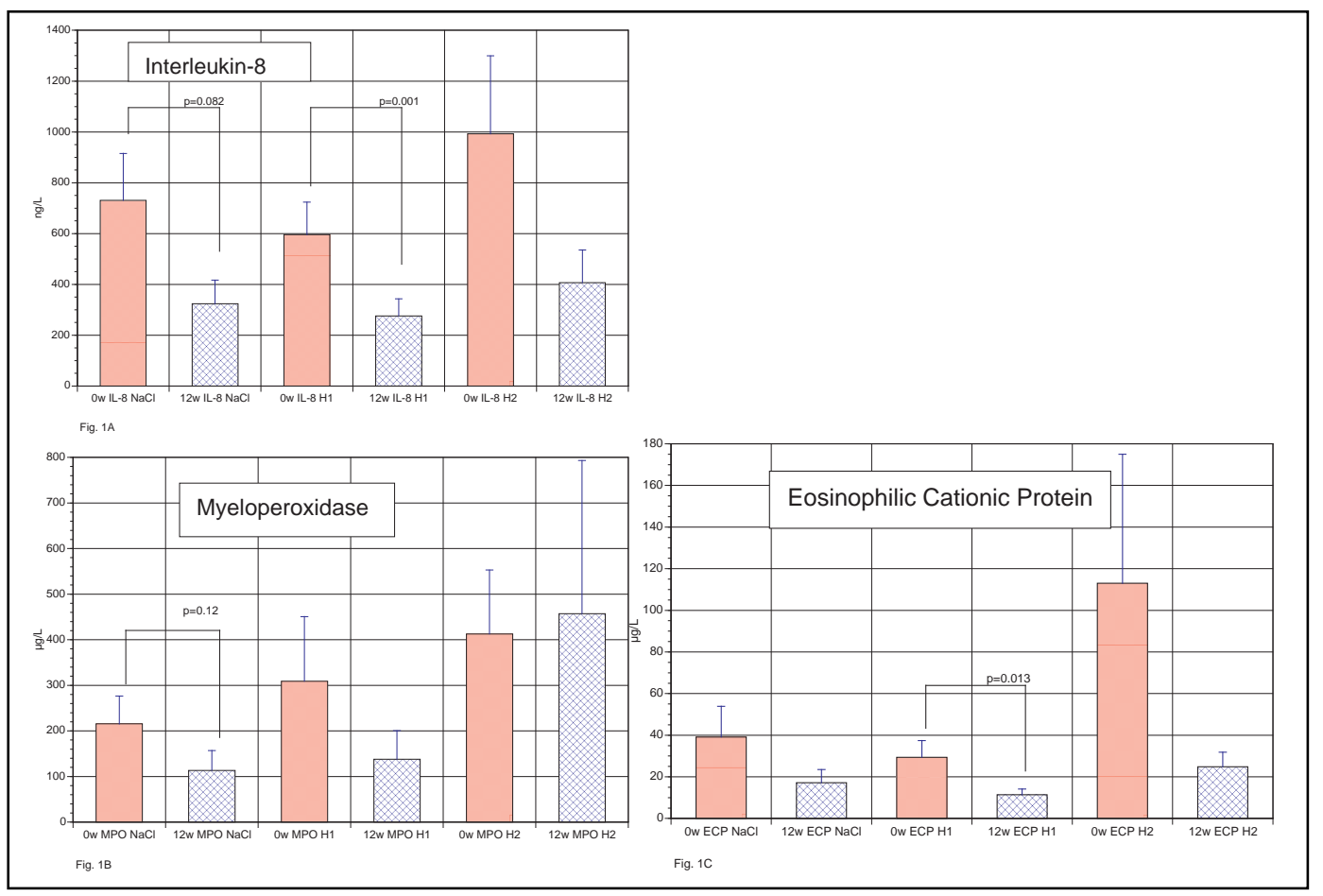

Fig. 1. Analytes in lavages with and without histamine before and late into the clarithromycin treatment. A. IL-8 was significantly reduced by clarithromycin at the low-dose histamine-observation $(\mathrm{p}<0.001)$. B. MPO was reduced by clarithromycin at the saline and low-dose histamine observations, but these changes failed to reach statistical significance. C. ECP was reduced by clarithromycin at the low-dose histamine observation $(\mathrm{p}<0.05)$. $(0 \mathrm{w}=$ start of the study, $12 \mathrm{w}=$ at the end of the study, $\mathrm{NaCl}=$ nasal lavage with saline, $\mathrm{H} 1=$ nasal lavage with histamine $40 \mu \mathrm{g} / \mathrm{ml}$ in saline, H2=nasal lavage with histamine $400 \mu \mathrm{g} / \mathrm{ml}$ in saline.) 


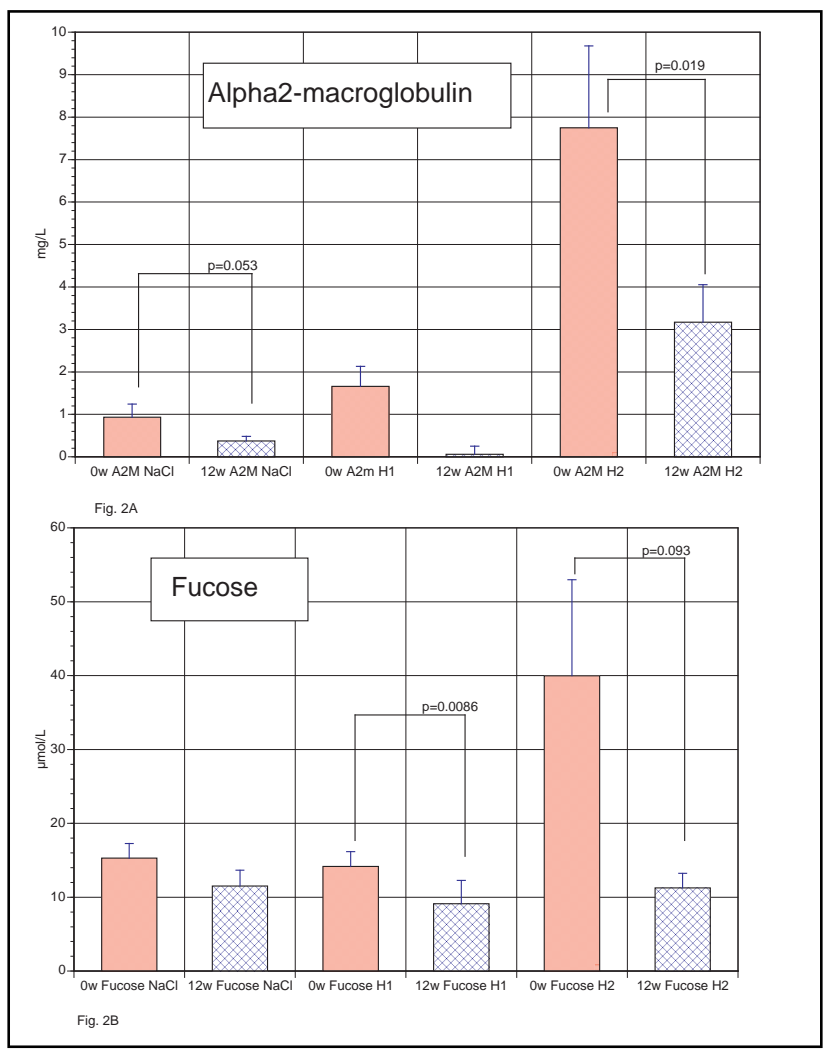

Fig. 2. Analytes in lavages with and without histamine before and late into the clarithromycin treatment. A. The saline lavage levels of $\alpha_{2}$-macroglobulin were reduced by clarithromycin $(\mathrm{p}=0.05)$. Furthermore, the exudative responsiveness to high-dose histamine was significantly reduced $(\mathrm{p}<0.05)$. B. Clarithromycin did not affect saline lavage levels of fucose. In contrast, clarithromycin attenuated the secretory responsiveness to histamine at the low-dose histamine observation $(\mathrm{p}<0.01)$. $(0 \mathrm{w}=$ start of the study, $12 \mathrm{w}=$ at the end of the study, $\mathrm{NaCl}=$ nasal lavage with saline, $\mathrm{H} 1=$ nasal lavage with histamine $40 \mu \mathrm{g} / \mathrm{ml}$ in saline, H2=nasal lavage with histamine $400 \mu \mathrm{g} / \mathrm{ml}$ in saline.) 


\section{References}

Castells MT, Ballesta J, Madrid JF, Aviles M and Martinez-Menarguez JA. Characterization of glycoconjugates in developing rat respiratory system by means of conventional and lectin histochemistry. Histochemistry (1991); 95: 419-426.

Cervin A, Kalm O, Sandkull $P$ and Lindberg S. One-year low-dose erythromycin treatment of persistent chronic sinusitis after sinus surgery: clinical outcome and effects on mucociliary parameters and nasal nitric oxide. Otolaryngol Head Neck Surg (2002); 126: 481-489.

Demoly P, Crampette L, Mondain M, Enander I, Jones I and Bousquet J. Myeloperoxidase and interleukin-8 levels in chronic sinusitis. Clin Exp Allergy (1997); 27: 672-675.

Erger RA and Casale TB. Interleukin-8 plays a significant role in IgE-mediated lung inflammation. Eur Respir J (1998); 11: 299-305.

Freney M, Irth H, Lindberg H, Alkner U, Greiff L, Persson C, Andersson M and Marko-Varga G. Rapid screening of airway secretions for fucose by parallel ligand-exchange chromatography with post-column derivatization and fluorescence detection. . Chromatographia (2001); 54: 439-445.

Greiff L, Andersson M, Akerlund A, Wollmer P, Svensson C, Alkner U and Persson CG. Microvascular exudative hyperresponsiveness in human coronavirusinduced common cold. Thorax (1994); 49: 121-127.

Greiff L, Andersson M, Coman WB, Lindberg H, Marko-Varga G, Wallwork B and Persson CG. Challenge-induced plasma exudation and mucinous secretion in human airways. Clin Physiol Funct Imaging (2005); 25: 241-245.

Greiff L, Andersson M, Erjefalt JS, Persson CG and Wollmer P. Airway microvascular extravasation and luminal entry of plasma. Clin Physiol Funct Imaging (2003); 23: 301-306.

Greiff L, Pipkorn U, Alkner $U$ and Persson CG. The 'nasal pool' device applies controlled concentrations of solutes on human nasal airway mucosa and samples its surface exudations/secretions. Clin Exp Allergy (1990); 20: 253259.

Greiff L, Venge $\mathrm{P}$, Andersson $\mathrm{M}$, Enander I, Linden M, Myint S and Persson CG. Effects of rhinovirus-induced common colds on granulocyte activity in allergic rhinitis. The J ournal of infection (2002); 45: 227-232.

Hamilos DL, Leung DY, Wood R, Meyers A, Stephens JK, Barkans J, Meng Q, Cunningham L, Bean DK, Kay $A B$ and et al. Chronic hyperplastic sinusitis: association of tissue eosinophilia with mRNA expression of granulocytemacrophage colony-stimulating factor and interleukin-3. J Allergy Clin Immunol (1993); 92: 39-48.

Ichimura K, Shimazaki Y, Ishibashi $T$ and Higo R. Effect of new macrolide roxithromycin upon nasal polyps associated with chronic sinusitis. Auris Nasus Larynx (1996); 23: 48-56.

MacLeod CM, Hamid QA, Cameron L, Tremblay C and Brisco W. Anti-inflammatory activity of clarithromycin in adults with chronically inflamed sinus mucosa. Adv Ther (2001); 18: 75-82.

Mariassy AT, Plopper CG, St George JA and Wilson DW. Tracheobronchial epithelium of the sheep: IV. Lectin histochemical characterization of secretory epithelial cells. The Anatomical record (1988); 222: 49-59.

Meyer P, Persson CG, Andersson M, Wollmer P, Linden M, Svensson C and Greiff L. Alpha2-macroglobulin and eosinophil cationic protein in the allergic airway mucosa in seasonal allergic rhinitis. Eur Respir J (1999); 13: 633-637.

Rogers DF. Airway goblet cells: responsive and adaptable front-line defenders. Eur Respir J (1994); 7: 1690-1706.

Rudack C, Stoll W and Bachert C. Cytokines in nasal polyposis, acute and chronic sinusitis. Am J Rhinol (1998); 12: 383-388. 
Suzuki H, Asada $\mathrm{Y}$, Ikeda K, Oshima T and Takasaka T. Inhibitory effect of erythromycin on interleukin-8 secretion from exudative cells in the nasal discharge of patients with chronic sinusitis. Laryngoscope (1999); 109: 407410.

Suzuki H, Shimomura A, Ikeda K, Furukawa M, Oshima T and Takasaka T. Inhibitory effect of macrolides on interleukin-8 secretion from cultured human nasal epithelial cells. Laryngoscope (1997); 107: 1661-1666.

Svensson C, Andersson M, Greiff L, Alkner $U$ and Persson CG. Exudative hyperresponsiveness of the airway microcirculation in seasonal allergic rhinitis. Clin Exp Allergy (1995); 25: 942-950.

Takeuchi K, Yuta A and Sakakura Y. Interleukin-8 gene expression in chronic sinusitis. Am J Otolaryngol (1995); 16: 98-102.

Takeuchi $\mathrm{N}$ and Ichimura $\mathrm{K}$. [Status of long-term administration of macrolide antibiotics in small dosages: a survey based on questionnaires]. Jpn J Antibiot (1997); 50 Suppl: 8-10.

Wallwork B, Coman W, Feron F, Mackay-Sim A and Cervin A. Clarithromycin and prednisolone inhibit cytokine production in chronic rhinosinusitis. Laryngoscope (2002); 112: 1827-1830.

Wallwork B, Coman W, Mackay-Sim A, Greiff L and Cervin A. A double-blind, randomized, placebo-controlled trial of macrolide in the treatment of chronic rhinosinusitis. Laryngoscope (2006); 116: 189-193.

Warringa RA, Mengelers HJ, Raaijmakers JA, Bruijnzeel PL and Koenderman L. Upregulation of formyl-peptide and interleukin-8-induced eosinophil chemotaxis in patients with allergic asthma. J Allergy Clin Immunol (1993); 91: 1198-1205.

Yamada T, Fujieda S, Mori S, Yamamoto $H$ and Saito H. Macrolide treatment decreased the size of nasal polyps and IL-8 levels in nasal lavage. Am J Rhinol (2000); 14: 143-148. 